\title{
A Double Layered Segmentation Algorithm for Cervical Cell Images based on GHFCM and ABC
}

\author{
G. Anna Lakshmi ${ }^{1}$ \\ ${ }^{1}$ Research Scholar, Manonmaniam Sundaranar University, Tirunelveli, India \\ Email: annalakshmi.msu@gmail.com \\ Dr. S. Ravi ${ }^{2}$ \\ ${ }^{2}$ Asst. Prof, Dept. of CSE, Pondicherry University, Pondicherry, India
}

Received: 26 May 2017; Accepted: 07 August 2017; Published: 08 November 2017

\begin{abstract}
Cancer is a life threatening disease and it engulfs the lives of many women. Due to the technology advancement, the medical science is drastically improved. A statistical report claims that the diagnostic decisions of radiologists show more false positive rates, which is very dangerous. However, when the radiologists are supported by computer aided applications, the false positive results are considerably reduced. Understanding the potentiality of computer aided applications, this paper presents a double layered segmentation algorithm for cervical cell images. The entire work is subdivided into three important phases, which are cervical image preprocessing, coarse and fine level segmentation. The preprocessing phase attempts to remove the noise and enhance the image quality by means of adaptive mean filter and Contrast Limited Adaptive Histogram Equalization (CLAHE) technique respectively. The coarse level segmentation process is achieved by Generalized Hierarchical Fuzzy C Means (GHFCM) and the fine level segmentation process is carried out by Artificial Bee Colony $(\mathrm{ABC})$ algorithm. The performance of the proposed segmentation algorithm is analysed in terms of accuracy, sensitivity and specificity. The experimental results show the efficacy of the proposed segmentation algorithm.
\end{abstract}

Index Terms-Cervical cancer, image segmentation, preprocessing, bio-inspired algorithm.

\section{INTRODUCTION}

Breast Cervical cancer is the fourth commonly occurring cancer among women worldwide and second common cancer in developing countries [1]. As on 2012, World Health Organization (WHO) has assessed that nearly 5,30,000 new cases have been affected by cervical cancer globally. The report added that about 1,22,844 women are diagnosed with cervical cancer in a year. However, the public shows least awareness with respect to this dreadful disease. This lack of knowledge about the cervical cancer results in increased mortality rates. The statistical report of the year 2012, states that over 67,477 patients die because of cervical cancer.
Unlike other cancers, cervical cancer does not show notable symptoms. Hence, periodical check ups alone can offer the women an ideal escape from this dreadful cancer. Owing to the advancement of medical science and technology, several equipments have been introduced to combat against cancer. The popular screening test for detecting cervical cell abnormality is the pap smear test. During the screening procedure, the healthcare professional takes the cervical cell samples followed by staining them. The process of staining is to easily differentiate between the cell components. It is for the healthcare professional to interpret the screen reports with high accuracy rates. However when the healthcare professional is assisted by a computerized system, the accuracy and sensitivity rates are improved. These improvements are because of the double layered inspection of the screen reports.

The advanced image processing techniques show its remarkable contributions towards detecting the abnormalities being present in the medical image. Understanding the necessity of an automated system to detect the abnormalities of cervical images, this work aims to present a system to segment the nuclei of the cervical cell images. However, achieving reasonable accuracy and sensitivity rates are not simple. This is because of the complex structure, poor contrast and unstable staining of the cervical cells. All these factors affect the accuracy rates of the abnormality detection system. Taking the mentioned challenges into account, this article presents an automated segmentation technique to extract nuclei from the cervical cells.

The entire work is categorized into three different phases, which are cervical image pre-processing, coarse and fine segmentation. The image pre-processing is the basic step, which makes the images suitable for the forthcoming processes such as segmentation, feature extraction and classification. In this work, the preprocessing step is concerned with noise elimination and contrast enhancement. The noise elimination is attained by the adaptive median filter and the contrast of the images is enhanced by CLAHE (Contrast Limited Adaptive Histogram Equalization) technique. The cervical image segmentation process is achieved in two 
steps and they are coarse and fine segmentation. The coarse segmentation process is achieved by the Generalized Hierarchical Fuzzy C Means (GHFCM). This level of segmentation roughly segments the image and the next level of segmentation refines the formed clusters. The fine segmentation process is achieved by Artificial Bee Colony (ABC) algorithm, which imitates the behaviour of honey bees.

This two level segmentation procedure produces better segmented images. The better the segmentation results, the easier is the classification procedure. The time consumption for the image classification process is very minimal, when the segmentation results are accurate. These points are taken into account and this work proposes a double layered segmentation technique. The highlights of this work are listed below.

- The noise elimination process is done by adaptive median filter, which conserves the edges and minute details of an image.

- The contrast of the images is enhanced by the CLAHE technique, which is a local contrast enhancement technique.

- The coarse level segmentation is carried out by GHFCM, which is efficient and robust.

- The fine level segmentation is done by $\mathrm{ABC}$ algorithm, which effectively refines the formed clusters.

- The double layered segmentation technique improves segmentation accuracy and sensitivity rates.

The remainder of this paper is organized as follows. The related literature with respect to image segmentation over cervical images is presented in section 2. The proposed cervical image segmentation technique is presented in section 3 . The performance of the proposed approach is analyzed in section 4. The concluding remarks of the proposed segmentation technique are presented in section 5 .

\section{RELATED WORK}

This section reviews the related literature with respect to the cervical image segmentation.

A method based on polar transformation is presented to detect and segment cervical cells in [2]. Silhouette technique is utilized to determine the number of clusters. However, this method involves computational complexity and consumes more time for image segmentation.

In [3], the cellular components such as nucleus and cytoplasm are segmented by means of Gaussian Mixture Models (GMM). This work intends to improve the segmentation accuracy by combining the GMM with shape based segmentation concept. The drawback of this work is the poor experimental analysis.

A segmentation algorithm based on Fuzzy C Means (FCM) and Back Propagation Neural Networks (BPNN) is proposed in [4]. The shape features are extracted from the image and fed as input to the BPNN. The regions are distinguished by the BPNN as nucleus or not.

In [5], the cervical cell segmentation is done by colour clustering method. Initially, the image is converted from RGB to HSV model, followed by which the segmentation is done. The segmentation of cell nucleus and cytoplasm is achieved by colour clustering.

A nuclei segmentation technique for cervical images is presented in [6]. The cervical images are segmented by mean-shift method. Initially, the contrast of the cervical images is improved by decorrelation stretch contrast enhancement technique. The process of segmentation is achieved by morphological operations.

In [7], the nuclei of the cervical images are segmented by an active adaptive contour modelling. The active contour modelling is constructed by morphological operations. The local regions of the image are extracted by Gaussian kernel function and split bregman technique performs the segmentation process.

A cervical cell image segmentation technique is proposed in [8], which involves nucleus enhancement and segmentation. The nucleus enhancement process is achieved by combining the colour and intensity information of the cervical images. The image is then reconstructed with respect to morphological patterns, in order to extract the regional maxima. The final segmentation process is performed by shape descriptors.

In [9], a watershed based segmentation technique is proposed for both cervical and breast cell images. Initially, a distance transform is applied over the image, followed by the application of H-minima transform. This transform is to segment the cells based on the distance. This method is found to be superior to the standard watershed technique.

A technique to segment the nucleus and cytoplasm in cervical cell images based on multi-scale convolutional network and graph partitioning approach is proposed in [10]. Initially, the multi-scale convolutional network extracts several scale-invariant features and performs rough segmentation. The graph partitioning approach improves the already formed clusters with the prior knowledge.

In [11], an automatic nuclei segmentation technique for cervical images is proposed. The cervical image is represented as by a Markov Random Field (MRF), on the basis of the watershed segmentation map. By this way, the lesions are detected and the segmentation is performed by means of loopy belief propagation over the MRF.

A GVF snake model based segmentation algorithm to segment the cervical images is presented in [12]. The proposed model intends to extract the nucleus from the cervical cells. The attributes of this model are set by the energy function.

In [13], a technique to segment the cervical cell nucleus is proposed. Initially, the clusters are formed by $\mathrm{k}$-means algorithm. This step is followed by the utilization of gradient vector flow snake model for extracting the gradient information of the object 
boundaries. The region is extracted by the object boundary and gray scale cluster.

A two-phase segmentation approach for cervical images is proposed in [14]. Initially, the cervical images are segmented by non-parametric hierarchical segmentation algorithm, which considers the spectral, shape and gradient information. The second phase extracts the nucleus and cytoplasm by taking the spectral and shape properties of the objects.

In [15], the cervical images are segmented by Intersecting Cortical Model (ICM). The attributes of ICM are optimized by Particle Swarm Optimization (PSO) algorithm. This results in better segmentation. The work proposed in [16] presents a segmentation algorithm for cervical images. This algorithm segments the cervical image by Radiating Gradient Vector Flow (RGVF) snake. The classification between different cervical cell classes is done by Support Vector Machine (SVM).

Motivated by the above works, this paper intends to present a double layered segmentation algorithm, which performs coarse and fine segmentation. The coarse segmentation is done by GHFCM and the fine segmentation process is carried out by the bio-inspired ABC algorithm. The proposed double layered segmentation approach is elaborated in the following section.

\section{Proposed Double Layered SEGMENTAtion ALGORITHM}

This section presents the detailed work flow of the proposed segmentation algorithm along with the overall flow of the work.

\section{A. Overall flow of the work}

This work intends to extract the nucleus of the abnormal cervical cells by means of double layered segmentation algorithm. However segmenting a cell is very challenging, owing to the complex structure of the cell, inconsistent cell staining and poor contrast. Thus, irrespective of the presence of several segmentation algorithms, there is still a constant demand for the accurate segmentation algorithm. This research work presents a double layered segmentation algorithm, in which two step segmentation process occurs.

The initial segmentation process presents coarse segmented cervical cells and the final segmentation process refines or enhances the existing segments of the cervical cells. This kind of segment refinement enhances the segmentation accuracy.

Besides this, the proposed segmentation procedure works fast, as well. The important phases of this work are cervical image pre-processing, coarse and fine segmentation. The pre-processing step achieves two different tasks, which are noise removal and contrast enhancement.

The noise being present in the cervical images is eliminated by the adaptive median filter. The contrast of the cervical images is improved by the CLAHE technique.
The overall process flow of the work is depicted in figure 1.

As soon as the cervical images are pre-processed, the images become fit for the forthcoming process. An effective pre-processing procedure maximizes the accuracy of any image analytic activity.



Fig.1. Overall flow of the work

Hence, in this work cervical image pre-processing process precedes the process of cervical image segmentation. The cervical image segmentation is carried out by two phases, which are coarse and fine segmentation. The coarse segmentation is done by GHFCM and the so formed clusters are refined by ABC algorithm. This double layered segmentation strives hard to arrive at accurate segmentation results. The working principle of the proposed approach is presented in the following sub-sections.

\section{B. Cervical Image Pre-Processing}

The pre-processing phase of cervical images is done in two steps. Initially, the noise is eliminated by employing adaptive median filter and the contrast of the images is enhanced by CLAHE technique. The adaptive median filter is utilized for denoising the cervical images, as this filter conserves the edges and minute information of the images. The noise removal and contrast enhancement processes are explained below.

\section{1) Noise removal by adaptive median filter}

The adaptive median filter denoises the cervical images through spatial processing. Each and every pixel is treated by adaptive median filter. The adaptive median filter operates by considering the neighbourhood pixels of every single pixel. The value of the manipulated pixel is matched against the values of the neighbourhood pixels.

In case of the unconnected value of the manipulated pixel with respect to the values of the neighbourhood pixels, the median value of the neighbourhood pixels is computed. The computed mean value is substituted in the place of the manipulated pixel. The major difference between the adaptive median filter and median filter is that the neighbourhood window size is fixed in median filter. 
On the other hand, the adaptive median filter does not allow fixed window size for noise removal process. The manipulated pixel always remains in the centre place of the window. In case, if the central pixel is observed to be affected by impulse noise, then the median value is substituted. Suppose, if the median value is found to be corrupted by impulse noise, then the size of the window is elongated. This process is repeated for all the pixels being present in an image. The resultant image is smoothened, while conserving the details of the image. The next step of cervical image pre-processing is to enhance the contrast of the image, which is presented as follows. The sample input images along with the sample pre-processed images are presented in figure 2.

\section{2) Cervical Image Contrast Enhancement by CLAHE}

The CLAHE is a local contrast enhancement technique, which intends to improve the image contrast. CLAHE enhances the brightness degree of an image, such that the details of an image can easily be located.

The colour dyes usually get smudged, which makes the object discrimination tougher. In order to process these images in a better way, contrast enhancement of the images is necessary. The CLAHE technique enhances the brightness of the images, however the images are saved from the issue of brightness saturation. Additionally, the CLAHE technique is very simple to implement, yet powerful [17-19].

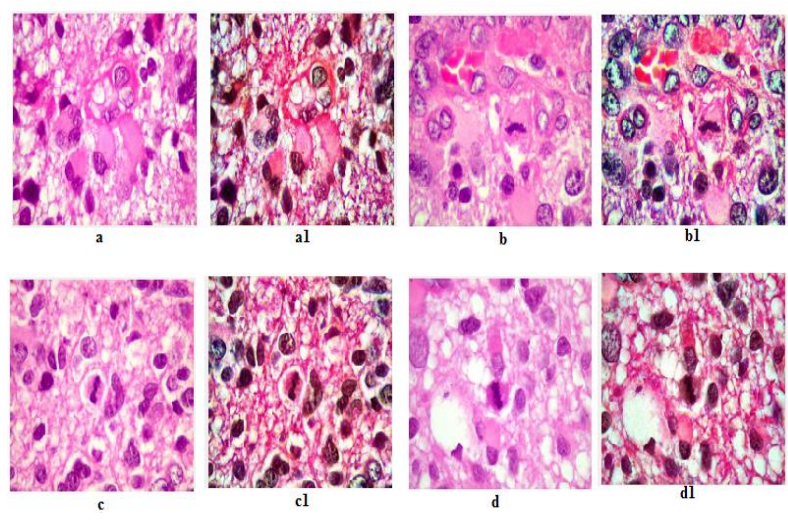

Fig.2. Pre-processed cervical images

In the above figure, image $a$ to $d$ are input images and $a l$ to $d l$ are the corresponding pre-processed images. As this work considers microscopic images of cervical cells, colour dyes are utilized to distinguish between the cell components.

\section{3) Coarse Segmentation by GHFCM}

This section intends to segment the pre-processed cervical images roughly. Usually, the segmentation procedure is carried out in an image for extracting the areas of interest. This work utilizes microscopic images and attempts to extract nucleus from the complex cell components. Understanding the complexity of nucleus extraction, this work performs a double layered segmentation.
The initial segmentation is achieved by GHFCM, which is the combination of Generalized Fuzzy C Means (GFCM) [20] and Hierarchical Fuzzy C Means (HFCM) [21]. GHFCM is employed for performing coarse level segmentation, as it is efficient and robust. In addition to this, the capability to withstand the noise makes it more suitable for this work [22].

The concept of Fuzzy C Means (FCM) segmentation is promising, because of its working principle. The information of the processing images is conserved by the FCM $[23,24]$. The traditional FCM algorithm is observed to be ineffective for noisy images, as it utilizes Euclidean distance measure.

The Euclidean distance measure does not work spatially and so the results are not impressive. Hence, this work employs auxiliary FCMs in the place of Euclidean distance. Besides this, the local spatial and component information of the images are combined together through generalized mean over fuzzy membership [25]. The GHFCM's objective function is presented below.

$$
O B_{f n}=\sum_{i=1}^{N} \sum_{c=1}^{C} \sum_{s c=1}^{S C} p_{i c}^{w} q_{i c s c}^{h} W_{f}|| m_{a}-\mu_{c s c} \|^{2}
$$

Where $i=\{1,2,3, \ldots, N\}$ and it denotes the number of images. $C$ is the total number of clusters and $S C$ is the total count of subclasses. The membership degree of $m_{i}$ in the $c^{\text {th }}$ cluster is denoted by $p_{i c}$ and $w$ is the weight component of the fuzzy membership function. $q_{i c s c}$ is the auxiliary membership function. $W_{f}$ is the weighing factor that manages the distance between the manipulated and the central pixel. $m_{a}$ indicates the intensity of an image and $\mu_{c s c}$ is the central point of the cluster. The computation of $p_{i c}, q_{i c s c}$ and $\mu_{c s c}$ is represented as follows.

$$
\begin{aligned}
p_{i c} & =\frac{\sum_{f \in N H W_{i}} w_{f} p_{d c}}{\sum_{p=1}^{C} \sum_{f \in N H w_{i}} w_{f} p_{d p}} \\
q_{i c s c} & =\frac{\sum_{f \in N H w_{i}} w_{f} q_{d c s c}}{\sum_{p=1}^{S C} \sum_{f \in N H w_{i}} w_{f} q_{d c s c}}
\end{aligned}
$$

The central point of the cluster $\mu_{c s c}$ is represented by the following equation.

$$
\mu_{c S c}=\frac{\sum_{i=1}^{N} \sum_{f \in N H W_{i}} p_{i c}^{w} q_{i c s c}^{h} m_{a}}{\sum_{i=1}^{N} p_{i c}^{w} q_{i c s c}^{h}}
$$

In the above equations, $N H W_{i}$ denotes the neighbourhood of pixel $i . m_{d c s c}$ denotes the distance function, which is the Euclidean distance.

By this way, the coarse segmentation is done by GHFCM. This algorithm considers the spatial relationship and distance function into account for performing segmentation. This initial step of segmentation is followed by the fine segmentation and is explained below.

\section{4) Fine Segmentation by ABC Algorithm}


$\mathrm{ABC}$ is a bio-inspired metaheuristic algorithm that intends to refine the already formed clusters. The ABC algorithm mimics the nature of honey bees and is proposed by Karaboga [26]. The major ingredients of ABC algorithm are food source, employed and unemployed bees. The goal of this algorithm is to find the best food source.

A food source is determined as the best by considering the distance, quality and the energy requirement of the bees. The distance between the bee hive and the food source must be minimal, such that the food source can be reached by the bees in a short span of time.

Secondly, the quality of the food source must be greater and the energy requirement of the bees for food consumption must be lower. Figure 3 depicts the coarse and fine segmented cervical images. The figures $a$ through $d$ are input images, $a l$ to $d l$ are coarsely segmented images and $a 2$ to $d 2$ are finely segmented images.

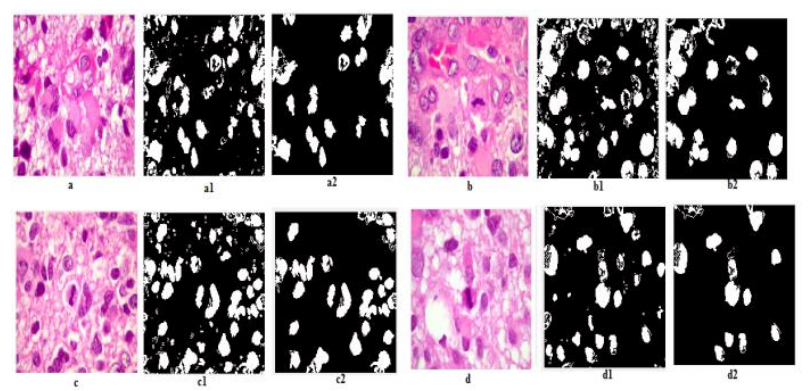

Fig.3. Coarse and fine segmented images

A swarm of bees are utilized for finding the best food source. The bee swarm is comprised of employed and unemployed bees. Employed bees cover the half portion of the swarm and the remaining bee swarm is occupied by unemployed bees. The main task of the employed bees is to find the information about the high quality food source and share the found information with the fellow bees.

Usually, the location and the distance information of the food source is shared with the fellow bees. There are two kinds of unemployed bees, which are scout and onlooker bees. The scout bees search for the new food source in the surrounding areas of the bee hive. The onlooker bees present in the bee hive and make the decision about the food source. The decision is made by considering the knowledge given by the employed bees.

The quality of the food source is assessed by the quantity of honey in the food source. The solution to the optimization issue is determined by the position of the food source. The quality of the food source acts as the fitness function of the algorithm. The working principle of the algorithm is that the food source search process is initiated by the scout bees.

The number of employed bees is equivalent to the number of food sources available. The employed and onlooker bees consume the food source continuously and so the amount of food source starts to reduce. In this scenario, the employed bees transform itself to scout bees to search for new food source. The proposed double layered segmentation algorithm is presented below.

\begin{tabular}{l} 
Double layered segmentation algorithm \\
\hline Input : Cervical cell images \\
Output: Nuclei segmented images \\
Begin \\
For images 1 to $n$ \\
Do \\
Remove noise by adaptive median filter; \\
Enhance the image contrast by CLAHE; \\
Perform coarse segmentation by GHFCM; \\
Save the results; \\
// Perform fine segmentation by ABC algorithm \\
Distribute the food source in a random fashion; \\
Compute the fitness value of the food source by eqn.5 \\
// Employed bee phase \\
Find new food source; \\
Compute fitness value of the food source; \\
Exploit GHFCM and greedy selection; \\
Compute the food source's probability by eqn. $7 ;$ \\
// Onlooker bee phase \\
Select a food source f $s_{i} ;$ \\
Compute the fitness value by eqn.6; \\
Exploit GHFCM and greedy selection; \\
Compare the old and new food source; \\
If (new food source is better) \\
Delete the old f $s_{i} ;$ \\
Save the best $f s_{i} ;$ \\
While termination condition is dissatisfied; \\
\hline
\end{tabular}

The classic ABC algorithm is organised into three stages and they are initialization, employed, onlooker and scout bees stages respectively. The initialization stage intends to set the control parameters and termination condition. The employed bees stage aims to search for the high quality food source in the surrounding location of the consumed food source. As soon as the new food source is found, the fitness of the food source is computed. This step is followed by the execution of greedy selection, which matches the quality of the older and the new food source. The knowledge being gained about the food source is shared with the onlooker bees.

The onlooker bee phase attempts to choose the best food source by considering the knowledge gained from the employed bees. Whenever a new food source is found, it is compared with the older one by means of greedy selection. At last, the employed bees start to become scout bees, when the optimal solutions could not be found. These stages are repeated till the optimal solution is reached [27, 28]. The proposed work involves double layered segmentation, because the convergence time of $\mathrm{ABC}$ is more. Hence, it would be better to pass the initial cluster point to the $\mathrm{ABC}$ and this is done by GHFCM.

Consider that $f s$ is the food source and is represented as $f s=1,2, \ldots, i$, which is a $\mathrm{N}$ dimensional vector. The value of $\mathrm{N}$ is represented by the total number of pixels and the segments. The initial count of pixels is two. The fitness value of the algorithm is given below. 


$$
f f=e_{v a r}+\max _{\mathrm{i}}\left(\text { var }_{\text {regi }}\right)+c(c s)
$$

In the above equation, $e_{v a r}$ is the variance of the edges in the coarse segmented image, $v a r_{r e g_{i}}$ is the variance of the $i^{\text {th }}$ region and $c(c s)$ is the cardinality of the connected components of the coarsely segmented images. As soon as the fitness of the food source is computed, the employed bees start to explore new food source in the surrounding region. On finding the new food source, the fitness of the new food source is computed and compared with the old one by greedy selection.

In case, if the similarity between the pixel and cluster centre similarity is more, then the older food source is deleted. This is represented as

$$
\operatorname{rec}_{i, j}=i p_{i, j}+\beta\left(i p_{i, j}-i p_{k, j}\right)
$$

In the above equation, $i p_{i, j}$ and $i p_{k, j}$ are the corresponding locations of the old and new pixels respectively. $\beta$ is the directionality that ranges from 1 to 1 . The food source is searched by changing the direction with respect to $\beta$. Thus, the related pixels are grouped together with respect to the cluster centre.

The bee searches the neighbourhood location for a new food source, by gradually changing the position. This step is followed by the computation of the probability of the food source, which is presented in the following equation.

$$
p r_{i}=\frac{f f_{i}}{\sum_{f s=1}^{i} f f_{f s}}
$$

The onlooker bees that reside in the bee hive selects the pixel on the basis of the probability value and strives to find some other pixel in the local neighbourhood. Thus, the onlooker bees select the pixel with optimal fitness value. The process continues until the best solution is obtained.

The following section analyses the performance of the proposed double layered segmentation algorithm. From figure 3, the capability of double layered segmentation is proved. The coarsely segmented images contain certain unwanted information and the fine segmentation procedure can segment the nuclei of the cervical cells efficiently.

\section{EXPERIMENTAL ANALYSIS}

The experimentation of this work is carried out in Matlab environment. This work randomly chooses hundred cervical images from the Herlev dataset [29]. The nuclei being present in the cervical cells are segmented by the double layered segmentation algorithm. The performance of the proposed segmentation algorithm is compared against the analogous segmentation approaches such as FCM+BPNN [4] and mean-shift based algorithm [6]. The experimental settings of the proposed algorithm are presented in table 1.
Table 1. Experimental Settings

\begin{tabular}{|c|c|}
\hline Experimental Parameters & Settings \\
\hline Population size & 35 \\
\hline $\begin{array}{c}\text { Chosen locations for neighbourhood } \\
\text { search }\end{array}$ & 14 \\
\hline Max iterations & 2000 \\
\hline
\end{tabular}

The performance metrics being considered are segmentation accuracy, sensitivity, specificity and time consumption. The formulae for computing these performance metrics are presented in the following equations.

$$
\begin{gathered}
a c c_{\text {seg }}=\frac{T_{p}+T_{n}}{T_{p}+T_{n}+F_{p}+F_{n}} \times 100 \\
S e n_{\text {seg }}=\frac{T_{p}}{T_{p}+F_{n}} \times 100 \\
S p_{\text {seg }}=\frac{T_{n}}{F_{p}+T_{n}} \times 100
\end{gathered}
$$

In the above equations, true positive, true negative, false positive and false negative rates are represented by TP, TN, FP and FN. Segmentation accuracy must be greater for any segmentation algorithm. This is because; the relevant pixels are grouped together, only when the segmentation accuracy is higher.

Sensitivity is the rate of perfectly segmented images to the total images that are correctly and incorrectly segmented. Specificity is the rate of images that are accurately segmented as cluster member to the sum of images that are correctly segmented as negative and the images that are wrongly segmented as the cluster member. The experimental results of the segmentation algorithms are compared and are presented below.

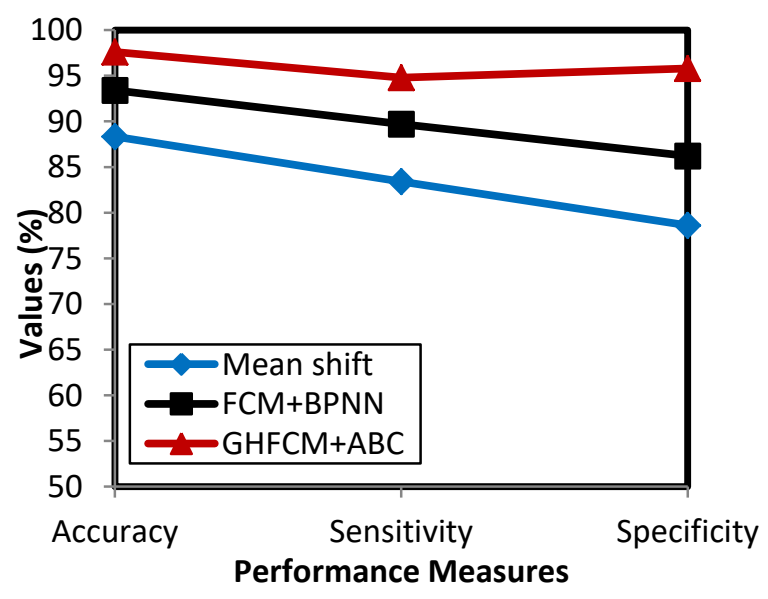

Fig.4. Comparative analysis on accuracy, sensitivity and specificity 


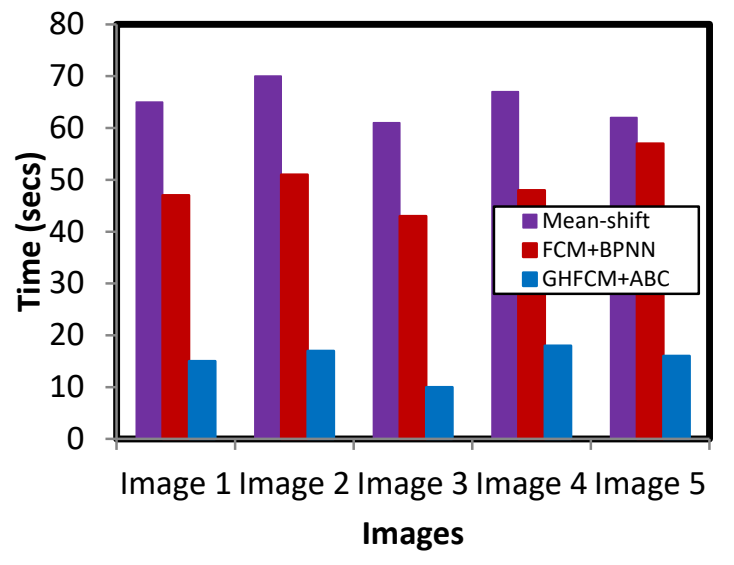

Fig.5. Time consumption analysis

From the experimental results, it is proved that the performance of the double layered segmentation algorithm is better than the analogous segmentation techniques. The accuracy of the proposed double layered segmentation algorithm is greater, because of the careful image pre-processing and the double layered segmentation steps. The maximum accuracy rate being shown by the proposed double layered segmentation algorithm is 97.6 .

The FCM+BPNN approach holds the second place with respect to accuracy rate with 93.4. The mean-shift based approach could prove only 88.34 percent.

On considering the sensitivity rates, the proposed approach proves the greatest value with 94.8 percent, which is followed by the value 89.7 percent and is shown by FCM+BPNN approach. The greatest specificity rate is proven by the proposed double layered segmentation approach, which is 95.8 percent. The combination of FCM and BPNN shows 86.2 as specificity rates and the mean-shift approach shows 78.6 percent. Thus, the combination of FCM and BPNN is the tough competitor to the proposed approach.

As far as the time consumption is considered, the time is measured in seconds. The time it takes to segment every single image is measured. Here, the graph is drawn for five sample cervical images. On comparison, this work observes that the time consumption to doublesegment the cervical images are comparatively lesser when compared to the existing approaches.

The maximum time being consumed by the proposed segmentation algorithm is 18 seconds and is for image 4 . The segmentation process for image 4 is completed in the shortest span of time, which is ten seconds. The combination of FCM and BPNN takes about fifty seven seconds to segment the image 5 at the maximum and forty three seconds are consumed for image 3 . The meanshift approach consumes more than a minute to process most of the images. On the whole, the proposed approach proves better time consumption rates.

The pre-processing step enhances the image quality, which plays a vital role in better segmentation. In addition to this, the proposed work does not rely on the single segmentation procedure but double layered segmentation procedure sharpens the performance of the algorithm.

The sensitivity and specificity rates are also satisfactory, when compared to the existing approaches. Time consumption is the most important metric of any algorithm, and so the time consumption of all the approaches are measured and compared. The time consumption of the proposed segmentation algorithm is quite lower than the comparative approaches.

\section{CONCLUSION}

This paper presents a double layered segmentation algorithm for cervical cell images. The images are preprocessed, so as to remove noise and enhance the image quality. The adaptive median filter and CLAHE technique are utilized for noise removal and contrast enhancement respectively. The segmentation process is carried out in two steps. Initially, the cervical images are segmented coarsely and then fine segmentation is carried out.

The coarse level segmentation is done by GHFCM and fine level segmentation is performed by ABC algorithm. The performance of the proposed segmentation algorithm outperforms the comparative algorithms. In future, it is planned to classify between the different kinds of cervical cells.

\section{REFERENCES}

[1] https://www.nhp.gov.in/disease/reproductivesystem/female-gynaecological-diseases-/cervical-cancer

[2] Neghina M, Rasche C, Ciuc M, Sultana A, Tiganesteanu C. Automatic detection of cervical cells in Pap-smear images using polar transform and k-means segmentation. InImage Processing Theory Tools and Applications (IPTA), 2016 6th International Conference on 2016 Dec 12 (pp. 1-6). IEEE.

[3] Ragothaman S, Narasimhan S, Basavaraj MG, Dewar R. Unsupervised segmentation of cervical cell images using Gaussian Mixture Model. InProceedings of the IEEE Conference on Computer Vision and Pattern Recognition Workshops 2016 (pp. 70-75).

[4] Sharma B, Mangat KK. An improved nucleus segmentation for cervical cell images using FCM clustering and BPNN. InAdvances in Computing, Communications and Informatics (ICACCI), 2016 International Conference on 2016 Sep 21 (pp. 1924-1929). IEEE.

[5] Cheng FH, Hsu NR. Automated cell nuclei segmentation from microscopic images of cervical smear. InApplied System Innovation (ICASI), 2016 International Conference on 2016 May 26 (pp. 1-4). IEEE.

[6] Agarwal P, Sao A, Bhavsar A. Mean-shift based segmentation of cell nuclei in cervical PAP-smear images. InComputer Vision, Pattern Recognition, Image Processing and Graphics (NCVPRIPG), 2015 Fifth National Conference on 2015 Dec 16 (pp. 1-4). IEEE.

[7] Zeng Z, Chen S, Tang S, Yin L. Unsupervised segmentation of cell nuclei in cervical smear images using active contour with adaptive local region fitting energy modelling. InBiomedical Engineering and Informatics (BMEI), 2015 8th International Conference on 2015 Oct 14 (pp. 250-254). IEEE. 
[8] Guan T, Zhou D, Fan W, Peng K, Xu C, Cai X. Nuclei enhancement and segmentation in color cervical smear images. InRobotics and Biomimetics (ROBIO), 2014 IEEE International Conference on 2014 Dec 5 (pp. 107112). IEEE

[9] Jung C, Kim C. Segmenting clustered nuclei using $\mathrm{H}$ minima transform-based marker extraction and contour parameterization. IEEE transactions on biomedical engineering. 2010 Oct;57(10):2600-4.

[10] Song Y, Zhang L, Chen S, Ni D, Lei B, Wang T. Accurate segmentation of cervical cytoplasm and nuclei based on multiscale convolutional network and graph partitioning. IEEE Transactions on Biomedical Engineering. 2015 Oct;62(10):2421-33.

[11] Alush A, Greenspan H, Goldberger J. Automated and interactive lesion detection and segmentation in uterine cervix images. IEEE Transactions on Medical Imaging. $2010 \mathrm{Feb}$;29(2):488-501.

[12] Zhang JW, Zhang SS, Yang GH, Huang DC, Zhu L, Gao DF. Adaptive segmentation of cervical smear image based on GVF Snake model. InMachine Learning and Cybernetics (ICMLC), 2013 International Conference on 2013 Jul 14 (Vol. 2, pp. 890-895). IEEE.

[13] Krishnan N, Sujatha SN. Segmentation of cervical cancer images using active contour models. InComputational Intelligence and Computing Research (ICCIC), 2010 IEEE International Conference on 2010 Dec 28 (pp. 1-8). IEEE.

[14] Kale A, Aksoy S. Segmentation of cervical cell images. InPattern Recognition (ICPR), 2010 20th International Conference on 2010 Aug 23 (pp. 2399-2402). IEEE.

[15] Tang JR, Isa NA, Ch'ng ES. Segmentation of cervical cell nucleus using Intersecting Cortical Model optimized by Particle Swarm Optimization. InControl System, Computing and Engineering (ICCSCE), 2015 IEEE International Conference on 2015 Nov 27 (pp. 111-116). IEEE.

[16] Sajeena TA, Jereesh AS. Automated cervical cancer detection through RGVF segmentation and SVM classification. In Computing and Network Communications (CoCoNet), 2015 International Conference on 2015 Dec 16 (pp. 663-669). IEEE.

[17] Andono PN, Purnama I, Hariadi M. UNDERWATER IMAGE ENHANCEMENT USING ADAPTIVE FILTERING FOR ENHANCED SIFT-BASED IMAGE MATCHING. Journal of Theoretical \& Applied Information Technology. 2013 Jun 30;52(3).

[18] Dhariwal S. Comparative analysis of various image enhancement techniques. International Journal of Electronics \& Communication Technology (IJECT). 2011 Sep;2(3):91-5.

[19] Kumar R, Suman HS. Comparative study of CLAHE DSIHE \& DHE schemes. International Journal of Research in Management, Science \& Technology.;1(1).

[20] Karayiannis, Nicolaos B. "Generalized fuzzy c-means algorithms." Fuzzy Systems, 1996., Proceedings of the Fifth IEEE International Conference on. Vol. 2. IEEE, 1996.

[21] Pedrycz, A. \& Reformat, M. Hierarchical FCM in a stepwise discovery of structure in data, Soft Computing, vol.10, no.3, pp: 244-256, 2006.

[22] Suman Shrestha, Image denoising using new adaptive based median filter, Signal \& Image Processing : An International Journal, Vol.5, No.4, pp.1-13, 2014.

[23] S. Chen and D. Zhang, Robust image segmentation using FCM with spatial constraints based on new Kernel- induced distance measure, IEEE Transactions on Systems, Man and Cybernetics 34(4) (2004), 1907-1916.

[24] D. Pham and J.L. Prince, An adaptive fuzzy c-means algorithm for image segmentation in the presence of intensity inhomogeneities, Pattern Recognit Lett 20 (1999), 57-68.

[25] Yuhui Zheng, Byeungwoo Jeon, Danhua Xu, Q.M. Jonathan $\mathrm{Wu}$ and Hui Zhang, Image segmentation by generalized hierarchical fuzzy C-means algorithm, Journal of Intelligent \& Fuzzy Systems 28, 961-973, 2015

[26] D. Karaboga, An idea based on honey bee swarm for numerical optimization, Technical Report-TR06, Erciyes University, Engineering Faculty, Computer Engineering Department, 2005.

[27] Dervis Karaboga, Beyza Gorkemli, Celal Ozturk, Nurhan Karaboga, A comprehensive survey: artificial bee colony (ABC) algorithm and applications, Artif Intell Rev, 2012.

[28] Dervis Karaboga, Celal Ozturk, A novel clustering approach: Artificial Bee Colony (ABC) algorithm, Applied Soft Computing 11 (2011) 652-657.

[29] http://mde-lab.aegean.gr/index.php/downloads

\section{Authors'Profiles}

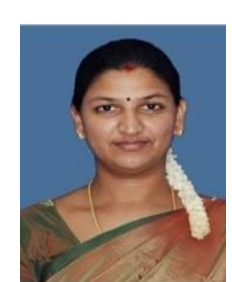

G.Anna Lakshmi has completed her $\mathrm{M}$. Tech in Computer Science and IT from CITE, Manonmaniam Sundaranar University, Tirunelveli, Tamilnadu, India. she has more than 14 years of teaching experience and guided handful of projects both at U.G and P.G level at various applications like Image Processing, Image retargeting, Web based application and wireless sensors. She is pursuing her Doctorate in Philosophy in Computer Science and Engineering at Manonmaniam Sundarnar University, Tirunelveli, Tamilnadu, India. She is presently working as Senior Professor in Department of Computer Science and Engineering, Kamaraj College of Engineering and Technology ,Virdhunagar, Tamilnadu , India Since 2008. Her research interest includes Medical Image Processing, Image Retargetting, Data Science $\&$ Data analytics wireless sensors. She is a Life member in ISTE, and Computer Society of India.

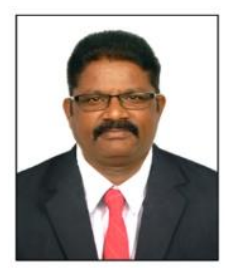

Dr. S. Ravi is now working as (Associate) Assistant Professor in the Department of Computer Science, School of Engineering and Technology, Pondicherry University, Pondicherry. He has 26 Years and 3 months of teaching experience and more than 11 years of research experience after his Post Graduate Degree in Technology. He has published more than 185 research papers in total, including 47 Scopus Indexed International Journals, 8 Springer Journal (Special Volume), 33 Other International Jouranals, 1 Elsvier Proceedia Computer Science, 7 Elsvier Journal Proceedings, 1 Springer Journal Proceedings, 1 IET Conference Proceedings, 1 IDES Conference Proceedings, 20 IEEE Conferences Proceedings, 42 International Conferences proceedings, 19 National Conferences Proceedings. He is a Senior Member of IEEE, Treasurer, IEEE SIPSHICOM, PODHIGAI Section, Member in IEEE Young Professionals, IEEE Computational Intelligence Society, IEEE Circuits and Systems Society, IEEE Sensors Council, IEEE Systems Council, IEEE Biometrics Council, IEEE Council on Electronic Design Automation, the 
Indian Science Congress and Life Member in Computer Society of India. His research interest includes Biometrics, Medical
Imaging, Skin Detection, Face Detection, Face Recognition, Face Tracking, Computer Vision, Digital Image Processing.

How to cite this paper: G. Anna Lakshmi, S. Ravi," A Double Layered Segmentation Algorithm for Cervical Cell Images based on GHFCM and ABC", International Journal of Image, Graphics and Signal Processing(IJIGSP), Vol.9, No.11, pp. 39-47, 2017.DOI: 10.5815/ijigsp.2017.11.05 